\title{
Fragmentation and clustering in star matter
}

\author{
F. Gulminelli
}
LPC, CNRS/IN2P3, Ensicaen, Université de Caen, F-14050 Caen cedex, France

\begin{abstract}
The specificity of the crust-core phase transition in neutron star at zero and finite temperature will be discussed. It will be shown that, as a consequence of the presence of long range Coulomb interactions, the equivalence of statistical ensembles is violated and a clusterised phase is expected which is not accessible in the grancanonical ensemble. A specific analytical Nuclear Statistical Equilibrium model will be presented and some new quantitative results relevant for the supernova dynamics will be shown. Finally, the analogies and differences with the phenomenon of nuclear fragmentation will be highlighted.
\end{abstract}

This is an Open Access article distributed under the terms of the Creative Commons Attribution License 2.0, which permits unrestricted use, distribution, and reproduction in any medium, provided the original work is properly cited. 